\title{
Erratum: Complete energy conversion between light beams carrying orbital angular momentum using coherent population trapping for a coherently driven double- $\Lambda$ atom-light-coupling scheme [Phys. Rev. A 100, 023811 (2019)]
}

\author{
Hamid Reza Hamedi @ , Emmanuel Paspalakis, Giedrius Žlabys, Gediminas Juzeliūnas, and Julius Ruseckas
}

(Received 2 July 2020; published 22 July 2020)

DOI: 10.1103/PhysRevA.102.019903

A number of errors have been detected in the published article. The $x$ axis of Figs. 3 and 4 should be scaled to $z /\left(40 L_{\mathrm{abs}}\right)$ rather than to $z / L_{\mathrm{abs}}$, so $L_{\mathrm{abs}}$ should be replaced with $40 L_{\mathrm{abs}}$ in all relevant discussions of these figures. Consequently, the total length of the medium becomes $L=1600 L_{\text {abs }}$ in the calculations of Figs. 3 and 4. Also, there should be $\bar{z}=80 L_{\text {abs }}$ and $z_{0}=800 L_{\text {abs }}$ in Fig. 3, and one has $\sigma=640 L_{\text {abs }}$ in Fig. 4 .

In Eq. (1), the $\hbar$ is missing and the single-photon detuning should be included for the probe fields so that

$$
H=\hbar \delta\left|e_{2}\right\rangle\left\langle e_{2}\right|-\hbar\left(\Omega_{p_{1}}\left|e_{2}\right\rangle\left\langle g_{1}\left|+\Omega_{p_{2}}\right| e_{2}\right\rangle\left\langle g_{2}\left|+\Omega_{c_{1}}\right| e_{1}\right\rangle\left\langle g_{1}\left|+\Omega_{c_{2}}\right| e_{1}\right\rangle\left\langle g_{2}\right|+\text { H.c. }\right),
$$

where we have assumed that the strong control fields are at single-photon resonance with each respective transition. Also, in the paragraph below Eq. (3), it should be $\delta_{1}=\delta_{2}=-\delta$.

In the density-matrix equations presented in the Appendix, one should add in appropriate places complex conjugations of the Rabi frequencies of the probe fields $\Omega_{p_{1}}$ and $\Omega_{p_{2}}$. Specifically, the second terms on the right-hand sides of Eqs. (A6)-(A8) should read $i \Omega_{p_{2}}^{*} \rho_{e_{2} g_{1}}, i \Omega_{p_{1}}^{*} \rho_{e_{2} g_{1}}-i \Omega_{p_{1}} \rho_{g_{1} e_{2}}$, and $i \Omega_{p_{2}}^{*} \rho_{e_{2} g_{2}}-i \Omega_{p_{2}} \rho_{g_{2} e_{2}}$, respectively. The first and second terms on the right-hand side of Eq. (A9) should read $i \Omega_{p_{1}} \rho_{g_{1} e_{2}}-i \Omega_{p_{1}}^{*} \rho_{e_{2} g_{1}}$ and $i \Omega_{p_{2}} \rho_{g_{2} e_{2}}-i \Omega_{p_{2}}^{*} \rho_{e_{2} g_{2}}$, respectively. Note that the Rabi frequencies of the control fields $\Omega_{c_{1}}$ and $\Omega_{c_{2}}$ are considered to be real quantities, so no complex conjugation is needed for them. Finally, below Eq. (A9) it should be $\Delta_{e_{2} g_{1}}=\Delta_{e_{2} g_{2}}=0$. We stress that the numerical calculations presented in Figs. 1-3 have been obtained with the correct form of the density-matrix equations.

The above corrections have no consequences on any of the discussed effects and do not affect the conclusions of the article.

We would like to thank Dr. D. Stefanatos for pointing out to the discrepancy in Figs. 3 and 4. 